Bangladesh J. Bot. 48(4): 1029-1035, 2019 (December)

\title{
THE NATURE OF PSII REACTIONS STABILITY UNDER OXIDATIVE STRESS
}

\author{
Jeyhuna Jafarova*, Rena Ganiyeva, Sona Bayramova \\ AND RALPHREED GaSANOv ${ }^{1}$ \\ Institute of Botany of National Academy of Sciences of Azerbaijan, \\ Baku, AZ1073, Azerbaijan
}

Keywords: Photosystem II, Reactive oxygen species (ROS), Photoinhibition, Methyl viologen, Na-ascorbate, Millisecond delayed fluorescence

\begin{abstract}
Comparative investigations of millisecond delayed fluorescence of chlorophyll $a$ (msec-DF Chl $a$ ) characteristics under oxidative stress induced separately by high intensity light and methyl viologen (MV) were investigated in pumpkin leaves (Cucurbita pepo L.). The inactivation of electron transport chain of PSII was observed in both experiments. The restoration of given characteristics observed at normal illumination with photoinhibited seedlings was found to enhance under action Na-ascorbate (Na-asc). The restoration of fluorescence characteristics on treated MV seedlings was recorded only at presence of Na-asc. Effect of Naasc to restore inactivated processes is considered to be due to its ability to actively neutralize reactive forms of oxygen generated under oxidative stress. It is suggested that mechanism leading to changes of induction pattern of msec-DF Chl $a$ of PSII has common nature in both cases.
\end{abstract}

\section{Introduction}

The abiotic stresses of environment have generated oxidative stress in plant cells. As a result of excitation of light reaction of photosynthesis the intensive production of reactive oxygen species (ROS) $\left({ }^{*} \mathrm{O}_{2}, \mathrm{O}_{2}{ }^{-*}, \mathrm{OH}^{-*}, \mathrm{H}_{2} \mathrm{O}_{2}\right)$ occur. Accumulation of ROS are unavoidable as a result of photosynthesis even under favourable conditions because the chloroplasts are potential source of oxygen toxic derivatives (Asada 1996). The damage of chloroplast components and decrease of photosynthesis activity start with inactivation of PSII, the most vulnerable link of photosynthetic apparatus (Bukhov 2004, Tyystjärvi et al. 2005, Anderson et al. 1996). Damage of oxygen evolving complex leads to denaturation and proteolysis of proteins, peroxidation of reaction centre lipids of PSII and suppression of electron transport in PSII (Biswal 2005, Biswal et al. 2011). Action of excess light on photosynthetic apparatus has been researched in detail, but it is not common opinion about photoinhibition mechanism (Tyystjärvi 2008, Vass 2012). Photoinhibition lead to inactivation of PSII due to damage of $\mathrm{D}_{1}$ protein involved in processes of formation of ROS (Andersson et al. 2001, Anja et al. 2011, Jafarova et al. 2012). Increasing of light intensity induce enhance formation oxidative components on donor side and reduce component on acceptor side of reaction transport chain of PSII that results in disruption of oxidation-reduction balance. Inactivation of PSII induced by donor side under photoinhibition took place because of noncoordinated transport of electrons from P680 and donation of electron from $\mathrm{Mn}_{4} \mathrm{O}_{5} \mathrm{Ca}$-claster on $\mathrm{RC}$ chlorophyll. The accumulation of long lived oxidizing radical $\mathrm{P}_{680^{+*}}$ and $\mathrm{Tyrz}^{+*}$ on donor side of PSII leads to degradation of $\mathrm{D}_{1}$ protein (Gasanov et al. 2007, Antal et al. 2009).

Inactivation of PSII by acceptor mechanism takes place as a result of stabilization of $\mathrm{Q}_{\mathrm{A}}{ }^{-}$by protonation (Tyystjärvi 2008, Hakala et al. 2006). At investigation oxidative stress as a chemical model is used MV as an oxidation mediator on acceptor side of PSI. Primary target of oxidative stress induced by MV are antioxidative enzymes sensitive to action of ROS (Sun et al. 2001).

*Author for correspondence: < j.jafarova@yahoo.com>. ${ }^{1}$ Baku State University, Baku, AZ1148, Azerbaijan. 
Also, superoxide is formed on acceptor side PSI, PSII particularly of OEC is more receptive to treatment by MV (Song et al. 2006). MV inhibited PSII by cyclization of electron flow through PSI, suppressing electron transport (Pavel 2009). Plant cells are protected against oxidative damage by broad spectrum of radical scavenging systems including antioxidant enzymes as well as non-enzymatic antioxidants, like glutathione and ascorbate. Ascorbic acid is considered as the most popular and powerful ROS-detoxifying compound because of its ability to donate electrons in a number of reactions (Nijs et al. 1991).

Ascorbate detoxifies $\mathrm{H}_{2} \mathrm{O}_{2}$ as electron donor to ascorbate reductase or $\mathrm{O}_{2}{ }^{-}$and ${ }^{\circ} \mathrm{OH}$ by nonenzymatic way and thus preventing enzymes inactivation.

At present most attention is devoted to kinetic investigatios of photosyntetic activity. The interaction of light and dark processes of photosynthesis is outstanding in induction processes when the transition of adapted to dark plants to stationary photosynthesis under action of excited light. For investigation of induction processes the delayed fluorescence of Chl $a$ related to PSII is used on photosynthetic objects (Gasanov et al. 2012). The delayed fluorescence method makes it possible to rapid test of plant stability to stress conditions and gives possibility to reveal most vulnerable part of electron transport chain. The method of msec-DF of Chl $a$ permits to track of restoration effect of antioxidants at stress situation that was aimed at the present work.

\section{Materials and Methods}

The study was conducted in Biophysics laboratory of the Botany İnstitute of Azerbaijan National Sciences Academy in 2016.

The leaves of 10-day seedlings of pumpkin (Cucurbita pepo L.) were grown as water culture in factorostate conditions at $22^{\circ} \mathrm{C}, 80 \%$ humidity and illumination $250 \mathrm{mkV} / \mathrm{sm}^{2}$.

For creation of oxidative stress due accumulation of ROS in chloroplasts the leaves were sprinkled by MV solution (1,1'-dimethyl-4,4'-bipyridinium dichloride) at concentration $100 \mu \mathrm{M}$; by means of high intensity light $\left(4000 \mu \mathrm{M}\right.$ photon $\left./ \mathrm{m}^{2} / \mathrm{s}\right)$ the photodamage of PSII was caused that characterised the stability of photosynthetic apparatus to oxidative stress, induced by photoinhibition. To protect from oxidative stress the low molecular compound Na-asc at concentration of $4 \cdot 10^{4} \mathrm{M}$ was used.

The functional state of photosynthetic apparatus was evaluated by means of delayed fluorescence of $\mathrm{Chl} a$ at millisecond time rang in vivo to be conditional on recombination processes in reaction centre of PSII (Gasanov et al. 2007, Goltsev et al. 2012). The kinetics of msec-DF of Chl $a$ was measured by phosphoroscope with time interval between excitation and luminescence equal $1,25 \mathrm{~ms}$.

\section{Results and Discussion}

In leaves incubated in MV solution the drop of intensity of fast (f.ph) and slow phase (sl.ph) of msec-DF of Chl $a$ was observed (Fig. 1). After $1 \mathrm{hr}$ treatment pumpkin leaves with MV solution the intensity of f.ph decreased by 3.6 times, sl.ph by 1.8 times relatively to control (Fig. 1A), after 2 hrs the intensity of f.ph were decreased by 4.4 times, sl.ph by 2.2 times (Fig. 1B). The rise of induction curves stationary phase (s.ph) by 1.6 times in relation to control in both variants were observed that gives reason to suppose violation in oxidation-reduction reactions at PSII chain (Fig. $1 \mathrm{~A}, \mathrm{~B})$.

To elucidate suppression mechanism of photosynthetic reaction in PSII the low molecular antioxidant Na-asc was used, its protected mechanism as it is known to be implemented by suppression of ROS formation (Sankhalkar et al. 2002). Action of Na-asc after $1 \mathrm{hr}$ leads to rise f.ph intensity by 2.5 times and sl.ph by 4.6 times relatively to MV action (Fig.1C). The intensity of both phases was decreased after 2 hrs (Fig.1D). 
The formation of highly oxidize radical pair $\mathrm{P} 60^{+}$and $\mathrm{TyrZ}^{+}$under stress is known to induce inactivation of donor side of PSII chain. The inactivation of acceptor side is known to take place due to accumulation of reduce first acceptor $\mathrm{Q}_{\mathrm{A}}$. Probably, action of MV inducing oxidative stress and accumulation of ROS have led to inactivation of electron transport chain of PSII. It is known that MV depressed the activity of antioxidant enzymes of superoxide dismutase and ascorbate peroxidase which are sensitive to action of ROS (Daisuke et al. 2016). The absence of phase reduction intensity during time exposition after action of MV are likely to be the result of ability of MV to self-oxidation on light, continuing to generate ROS. That is why protective effect of Naasc appears after $1 \mathrm{hr}$ MV action was weakened to $2 \mathrm{hrs}$.

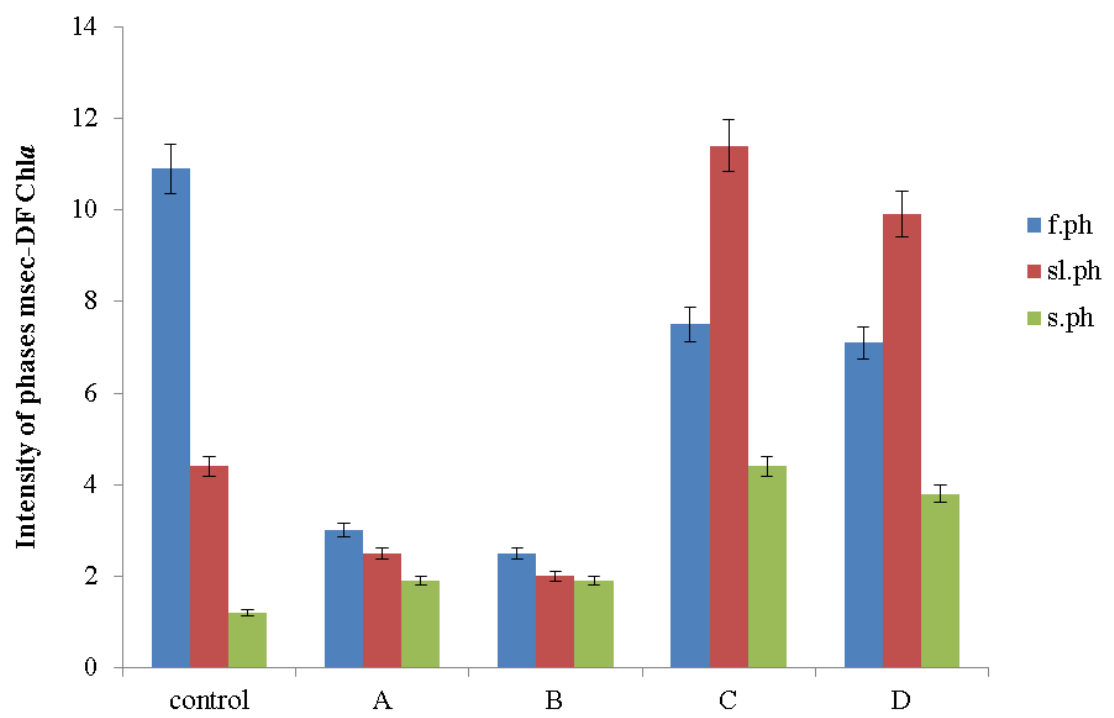

Fig.1. Results of induction of msec-DF Chl $a$ changes of pumpkin leaves seedlings treated by MV solution at concentration $100 \mu \mathrm{M}$. Intensity of phase of msec-DF Chl $a$ : fast (f.ph), slow (sl.ph), stationary phase (s.ph) were conducted 30 min after incubation of leaves in MV after 1 (A), 2 hrs (B) and after action of Na-asc solution in concentration $4 \cdot 10^{4} \mathrm{M}$ on treated by MV leaves also after 1 (C) and $2 \mathrm{hrs}$ (D).

By means of objective analysis of equilibrium between acceptor and donor side of electron transfer chain of PS II the changes of relation value f.ph. and sl.ph to s.ph ( f.ph/s.ph: sl.ph/s.ph) were observed (Table 1). After 1 and 2 hrs treatment of leaves by MV the drop of these values relative to control was observed. The value of f.ph decreased by 5.8 times after $1 \mathrm{hr}$ and the value of sl.ph by 3 times, after 2 hrs by 7 and 3 times. Action of Na-asc was shown to restored given values and f.ph/s.ph increased by 1.5 times and value sl.ph/s.ph by 2.5 times relatively to action of MV.

The observed rise of stationary level on induction curve is considered to be associated in condition with MV Na-asc did not can fully support oxidation-reduction balance between photosystems and fully neutralized superoxide radical (life time $\sim 2 \mathrm{~ms}$ ) which has time to migrate to surrounding of PSII. Displacement of redox state enhanced of electron flow PSI resulting in increase of s.ph level and according decreasing value of ratio to stationary phases.

After treatment of seedlings of pumpkin during $2 \mathrm{hrs}$ by light of high intensity the changes of phases msec-DF Chl $a$ was measured (Fig. 2). The drop of f.ph intensity relatively to control up to 4.2 times was registered. The slow phase almost did not register and was by 10 times lower 
relatively to control. Photoinhibited seedlings were transferred on definite time to normal illumination and restoration phase of msec-DF Chl $a$ were observed (Fig. 2). After 1.5 of light exposition the partial restoration of f.ph intensity it was observed that it increased by 1.6 times, intensity of sl.ph increased by 1.5 times higher relatively photoinhibition (Fig. 2). After exposition of seedlings on normal illumination during 5 hrs more greater restoration of intensities as f.ph (by 3.4 times), so sl.ph (by 9 times) that becomes nearly equal to control (Fig. 2). The stationary phase (s.ph) level on induction curve in both variants was equal to control and only in variant of seedling exposition during $5 \mathrm{hrs}$ its insignificant rise (by 1.4 times) was observed (Fig. 2).

Table 1. Changes of value of relation f.ph and sl.ph to s.ph (f.ph/s.ph and sl.ph/s.ph) of msec-DF Chl $a$ after incubation of pumpkin seedlings leaves in MV solution for 1 and 2 hrs without and with treatment by Na-asc after 1 and 2 hrs of restoration.

\begin{tabular}{lccccc}
\hline Msec-DF Chl $a$ & Control & \multicolumn{2}{c}{ MV $(100 \mu \mathrm{M})$} & \multicolumn{2}{c}{ Na-asc $\left(4 \bullet 10^{-4} \mathrm{M}\right)$} \\
\cline { 3 - 6 } & & $1 \mathrm{~h}$ & $2 \mathrm{~h}$ & $1 \mathrm{~h}$ & $2 \mathrm{~h}$ \\
\hline f.ph/s.ph & 9.2 & 1.6 & 1.3 & 1.7 & 1.9 \\
\hline sl.ph/s.ph & 3.6 & 1.3 & 1.05 & 2.6 & 2.6 \\
\hline
\end{tabular}

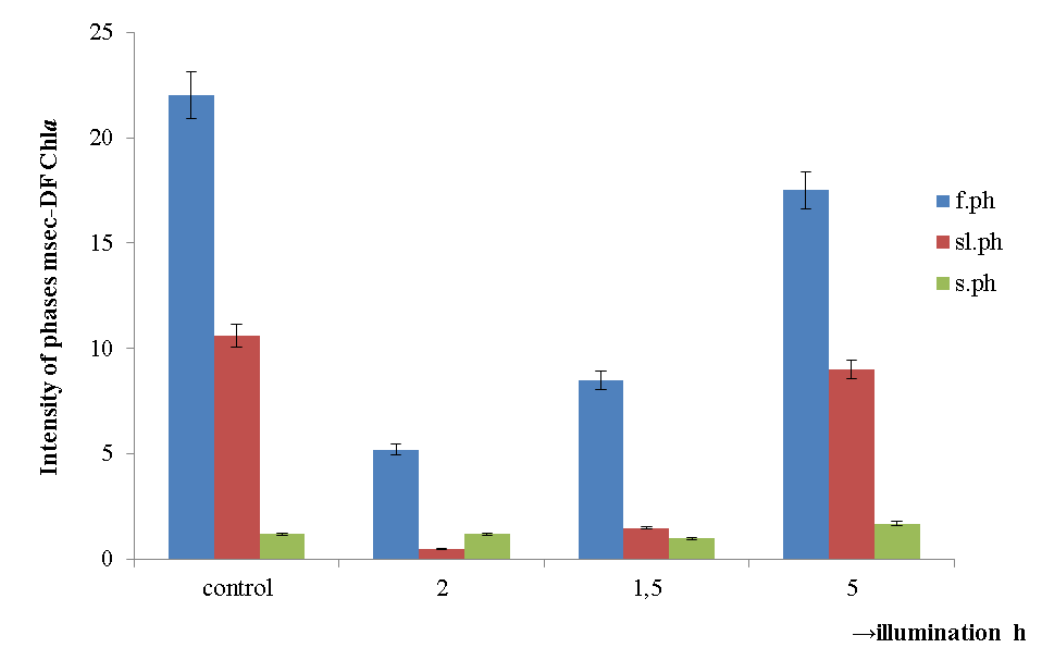

Fig. 2. The results of changes of induction character of msec-DF Chl $a$ of pumpkin seedlings leaves subjected to action of high intensity light $\left(4000 \mu \mathrm{M}\right.$ photons $\left./ \mathrm{m}^{2} / \mathrm{s}\right)$ during $2 \mathrm{hrs}$ (photoinhibition of PSII) and transferred to normal illumination $\left(250 \mathrm{mkV} / \mathrm{sm}^{2}\right)$. The changes of phase intensity were determined: fast (f.ph), slow (sl.ph), stationary (s.ph) after 1.5 and 5 hrs of restoration.

The uncoordinated transport of electron transport chain of PSII observed during photoinhibition partly was restored dependently from exposition time under transfer of plants to normal illumination. Under treatment of seedlings by Na-asc the restoration of millisecond intensity of phases took place significantly (Fig.3). The intensity f.ph and sl.ph were restored after $1.5 \mathrm{hr}$ and was equal to 2.3 and 9 times, after $5 \mathrm{hrs}$ by 2.4 and 14 higher relatively to photoinhibition. s.ph remained on control level in all variants (Fig. 3). 


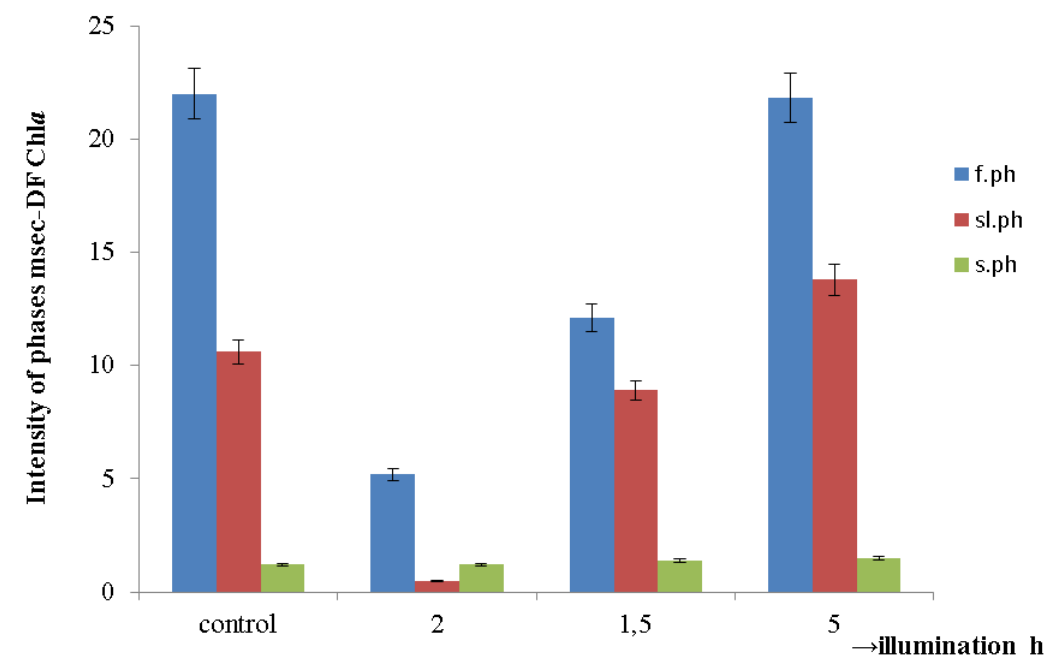

Fig. 3. The results of induction character changes of msec-DF Chl $a$ of pumpkin seedlings leaves subjected to action of high intensity light $\left(4000 \mu \mathrm{M}\right.$ photons $\left./ \mathrm{m}^{2} / \mathrm{s}\right)$ during $2 \mathrm{hrs}$ (photoinhibition of PSII) and transferred after a treatment by Na-asc photoinhibited seedlings to normal illumination $\left(250 \mathrm{mkV} / \mathrm{sm}^{2}\right)$. The changes of phase intensity were determined: fast (f.ph), slow (sl.ph), stationary (s.ph) after 1.5 and $5 \mathrm{hrs}$ of restoration.

Action of Na-asc under photoinhibition enhanced reduction of fluorescence activity characteristic during time exposition. ROS, involved to photoinhibition drive to $\mathrm{D}_{1}$ protein degradation slowly than the PSII inactivation. ROS has inhibited synthesis the protein $\mathrm{D}_{1}$ de novo (Nishiyama et al. 2015).

The restoration of values f.ph/sl.ph and s.ph/sl.ph observed after photoinhibition (after $1.5 \mathrm{hr}$ by 2 and 7.5 times; after 5 hrs by 2.4 and 2.6 times, correspondingly) was found to increase in the presence of Na-asc (Table 2). After $1.5 \mathrm{hrs}$ action of Na-asc values f.ph/s.ph restored by 2 times, after $5 \mathrm{hrs}$ by 3.4 times. Restoration of sl.ph/s.ph value was more intensive and to $5 \mathrm{hrs}$ exceed of control variant.

Table 2. The changes of relation values of f.ph. and sl.ph to s.ph of msec-DF Chl $a$ in leaves seedling of pumpkin photoinhibited during $2 \mathrm{hrs}\left(4000 \mu \mathrm{M}\right.$ photons $\left./ \mathrm{m}^{2} / \mathrm{s}\right)$ and then transferred to normal illumination $\left(250 \mathrm{mkV} / \mathrm{sm}^{2}\right)$ on 1.5 and $5 \mathrm{hrs}$ after treatment by Na-asc.

\begin{tabular}{|c|c|c|c|c|c|c|}
\hline \multirow[t]{2}{*}{$\begin{array}{l}\text { Msec-DF } \\
\text { Chl } a\end{array}$} & \multirow[t]{2}{*}{ Control } & \multirow[t]{2}{*}{$\begin{array}{c}2 \mathrm{hrs} \text { photoinhibition } \\
\left(4000 \mu \mathrm{M} \text { photons } / \mathrm{m}^{2} / \mathrm{s}\right)\end{array}$} & \multicolumn{2}{|c|}{$\begin{array}{c}\text { Normal illumination } \\
\left(250 \mathrm{mkV} / \mathrm{sm}^{2}\right)\end{array}$} & \multicolumn{2}{|c|}{$\begin{array}{l}\text { Normal illumination } \\
\left(250 \mathrm{mkV} / \mathrm{sm}^{2}\right) \mathrm{Na} \text {-asc }\end{array}$} \\
\hline & & & $1.5 \mathrm{~h}$ & $5 \mathrm{~h}$ & $1.5 \mathrm{~h}$ & $5 \mathrm{~h}$ \\
\hline f.ph/s.ph & 18,3 & 4,3 & 8.5 & 10.3 & 8.6 & 14.5 \\
\hline sl.ph/s.ph & 8,8 & 0,2 & 1.5 & 5,3 & 6.4 & 9.2 \\
\hline
\end{tabular}

Restoration by Na-asc processes suppressed by photoinhibition and resulted by action of MV took place in induction period of msec-DF Chl $a$ and appeared to be associated with result effective neutralization formed ROS.

So, it may be suggeted that mechanism leading to change of induction picture of DF Chl $a$ as a results of action of both factors has common nature. One of the key ways to enhance resistence 
of photosynthetic apparatus to stress under action of protective system of plant is generally accepted to increase antioxidant enzymes activity and/or the low molecular antioxidant action effectivity. The photosynthetic apparatus switched over to adaptive program and thus is providing its resistance to stress.

\section{References}

Asada K 1996. Radical production and scavenging in the chloroplasts. In: Photosynthesis and the Environments 128-150.

Anderson B and Barber J 1996. Mechanisms of photodamage and protein degradation during photoinhibition of photosystem II. In: Baker NR, Ed. Photosynthesis and the environment. Dordrecht, The Netherlands: Kluwer Academic Publishers pp. 101-121.

Andersson B, Aro EM and Ohad I 2001. The redox state of the plastoquinone pool controls the level of the light-harvesting chlorophyll a/b binding protein complex II (LHC II) during photoacclimation. Photosynth Res. 68(2): 163-74.

Anja Krieger-Liszkaya, Peter BK and Eva H 2011. Superoxide anion radicals generated by methylviologen in photosystem I damage photosystem II. Physiologia Plantarum 142: 17-25.

Antal T.K, Lo W, Armstrong WH and Tyystjärvi E 2009. Illumination with ultraviolet or visible light induces chemical changes in the water-soluble manganese complex, [Mn(4) O (6)(bpea) (4)] $\mathrm{Br}(4)$. Photochem Photobiol. 85(3): 663-8.

Bukhov NG 2004. Dynamic light regulation of photosynthesis. Plant Physiol. 51(6): 825-837.

Biswal B 2005. Photosynthetic response of green plants to environmental stress: Inhibition of photosynthesis and adaptational of photosynthesis and adaptational mechanisms. In: Handbook of Photosynthesis. Pessarakli M (Ed.), $2^{\text {nd }}$ Edth., CRC Press. Florida, pp. 739-749.

Biswal B, Joshi PN, Raval MK and Biswal UC 2011. Photosyntesis, a global sensor of environmental stress in green plants: stress signaling and adaptation. Current Sci. 101: 47-56.

Daisuke T, Takumi S, Hashiguchi M, Sejima T and Miyake Ch 2016. Superoxide and singlet oxygen produced within the thylakoid membranes both cause photosystem I photoinhibition. Plant Physiol. 171(3): 1626-1634.

Gasanov RA, Alieva S, Arao S, Ismailova A, Katsuta N, Kitade H, Yamada S, Kawamori A and Mamedov F 2007. Comparative study of the water oxidizing reactions and the millisecond delayed chlorophyll fluorescence in photosystem II at different pH. Photochem. Photobiol. 86: 160-164.

Gasanov RA, Aliyeva SA and Mammedov F 2012. Delayed fluorescence in a millisecond range - A probe for donor side-induced photoinhibition in photosystem. Overviews on recent progress and future perspectives. In: Guruprasad KN, İtoh S and Mohanty P (Ed.) Narosa Publising House, New Dehli, 7: $101-107$.

Goltsev V, Zaharieva I, Chernev P and Strasser R.J 2009. Delayed chlorophyll fluorescence as a monitor for physiological state of photosynthetic apparatus. Biotechnol. Equip. 23: 452-457.

Hakala M, Rantamäki S, Puputti EM, Tyystjärvi T and Tyystjärvi E 2006. Photoinhibition of manganese enzymes: Insights into the mechanism of photosystem II photoinhibition. J. Exp. Bot. 57(8): 1809-16.

Jafarova J, Bayramova S and Ganiyeva R 2012. The role of ascorbate in protection of PSII from photooxidative stress in cucumber (Cucumis sativus L.) leaves. - Xth International scientific conference materials "Introduction of non-traditional and rare plants" 1: 215-220.

Nijs D and Kelley PM 1991. Vitamins C and E donate single hydrogen atoms in vivo. FEBS Lett. 284: 147151.

Nishiyama Y, Yamamoto H, Allakhverdiev SI, Inaba M, Yokota A and Murata N 2015. Oxidative stress inhibits the repair of photodamage to the photosynthetic machinery EMBO J. 20(20): 587-94.

Pavel P 2009. Production of reactive oxygen species by photosystem II. Biochim. Biophys. Acta (BBA) Bioenergetics 1787(10): 1151-1160.

Sankhalkar S and Sharma PK 2002. Protection against photooxidative damage provided by enzymatic and non-enzymatic antioxidant system in sorghum seedlings. Indian J. Exp. Biol. 40(11): 1260-8.

Sun MC, Kye HS, Jae-Sung K and Youn-II P 2001. Inactivation of photosystem I in cucumber leaves exposed to Paraquat-induced oxidative stress. J. Photo. Science 8(1): 13-17. 
Song XS, Lan TC, Shi K, Mao WH, Ogweno JO, Zhou YH and Yu JQ 2006. The response of antioxidant enzymes in cellular organelles in cucumber (Cucumis sativus L.) leaves to methyl viologen-induced photo-oxidative stress. Plant Growth Regul. 49: 85-93.

Tyystjärvi E, Hakala M and Sarvikas P 2005. Mathematical modelling of the light response curve of photoinhibition of photosystem II. Photosynthesis Research 84: 1, 21-27.

Tyystjärvi E 2008. Photoinhibition of photosystem II and photodamage of the oxygen evolving manganese cluster. Coord Chem. Rev. 252: 361-376.

Vass I 2012. Molecular mechanisms of photodamage in the photosystem II complex. Biochim. Biophys. Acta 1817(1): 209-17.

(Manuscript received on 16 March, 2018; revised on 12 April, 2019) 